Horizons philosophiques

\title{
La conscience de l'absurdité chez Camus
}

\section{L'héritage d'un autre existentialisme}

\section{Adelino Braz}

Volume 16, numéro 2, printemps 2006

Héritage et réception de la pensée existentialiste

URI : https://id.erudit.org/iderudit/801315ar

DOI : https://doi.org/10.7202/801315ar

Aller au sommaire du numéro

Éditeur(s)

Collège Édouard-Montpetit

ISSN

1181-9227 (imprimé)

1920-2954 (numérique)

Découvrir la revue

Citer cet article

Braz, A. (2006). La conscience de l'absurdité chez Camus : l'héritage d'un autre existentialisme. Horizons philosophiques, 16(2), 1-8.

https://doi.org/10.7202/801315ar d'utilisation que vous pouvez consulter en ligne.

https://apropos.erudit.org/fr/usagers/politique-dutilisation/ 


\section{La conscience de l'absurdité chez Camus}

\section{l'héritage d'un autre existentialisme}

Dans un entretien publié en 1945 dans Les nouvelles littéraires, Albert Camus souligne avec une vigoureuse insistance sa démarcation par rapport à la philosophie existentialiste telle qu'elle est incarnée par Jean-Paul Sartre : «Non, je ne suis pas existentialiste ${ }^{1}$. Les raisons de cette position sont doubles : d'une part, l'auteur de l'Étranger considère qu'il n'est pas philosophe, et en ce sens, il ne croit pas suffisamment en la raison pour donner son assentiment à un quelconque système de pensée. D'autre part, l'existentialisme a pour effet de proposer soit une nouvelle divinité, soit une divination de l'histoire, solutions qui ne conviennent guère à une pensée qui cherche à déterminer entre ces deux horizons, une vérité qui soit supportable. "Je comprends bien l'intérêt de la solution religieuse, précise Camus, et je perçois très particulièrement l'importance de l'histoire. Mais je ne crois ni à l'une ni à l'autre au sens absolu2". Face à une telle position, nous sommes donc obligés d'admettre que la réflexion sur le sens de l'existence diffère radicalement des thèses existentialistes, qu'elles soient portées sur Dieu ou bien athées.

Toutefois, il est important d'apporter ici une nuance. Malgré cette prise de position, il est difficile de situer l'écart revendiqué par Camus, surtout lorsqu'il écrit dans Le mythe de Sisyphe «l'individu ne peut rien et pourtant il peut tout ${ }^{3}$ ", formule qui s'inscrit dans cette morale d'action et d'engagement inhérente à l'existentialisme : "L'homme étant ce dépassement et ne saisissant les objets que par rapport à ce dépassement, est au cœur, au centre de ce dépassement ${ }^{4} »$, suggère Sartre.

Or, il ne s'agit pas pour Camus de refuser sa priorité à l'affirmation de l'existence mais bien plutôt de renoncer à un certain système de pensée sur l'existence. Dans son dernier entretien, Camus apporte une nuance d'importance par rapport à sa critique. Si les prémisses de l'existentialisme se trouvent, comme il le croit, chez Pascal, Kierkegaard, Nietzsche, ou Chestov, alors sa réflexion n'est qu'un héritage de l'existentialisme. Au contraire, si ces prémisses se situent dans la pensée de " nos existentialistes5", et notamment de Sartre, alors il existe une contradiction avec son propre mode de penser le monde. Par conséquent, il s'agit bien de déterminer en quel sens 
Camus se revendique d'un certain existentialisme, autre que celui de ses contemporains. En ce sens, la question essentielle de notre réflexion consiste à cerner les prémisses fondamentales d'une pensée sur l'existence qui ne se confond pas avec le discours existentialiste. L'enjeu de cette question est bien de marquer l'écart entre l'existence comme donnée et l'existence comme pensée. À ce titre, il convient d'entreprendre une relecture de deux textes essentiels, Le mythe de Sisyphe et l'Homme révolté.

Avant de cibler rigoureusement les points de désaccords explicites formulés par Camus à l'égard de l'existentialisme, il convient d'en reprendre les thèses essentielles telles qu'elles sont présentées par Sartre dans L'existentialisme est un humanisme. Le point de départ déroutant de cette pensée est de montrer à quel point la vie en elle-même n'a pas de sens a priori 6 . En effet, contre Leibniz notamment qui pose l'existence d'une substance qui contient en ellemême toute sa notion, autrement dit qui tire d'elle-même l'ensemble de tous ses actes à venir selon une harmonie préétablie par un décret divin 7 , Sartre considère que l'homme est un être ouvert à l'existence au sens où son existence ne répond à aucune détermination ontologique.

Cela pose du coup deux conséquences majeures : en premier lieu, il faut admettre que la liberté est alors un donné pour l'homme dans la mesure où il n'existe aucune essence prédéfinie. L'homme au début n'est rien, il a à se faire et cela continuellement. Ceci implique du coup qu'il est lui-même son propre législateur et que dans une certaine mesure il est pleinement autonome. C'est pourquoi sa tâche majeure consiste de façon paradoxale à donner un sens à une vie qui en soi n'en a aucun. L'univers humain est donc celui de la seule subjectivité vécue comme réalité indépassable. A partir de ce point, Sartre distingue, comme le fera Camus également, deux espèces d'existentialisme : d'une part, ceux qui trouvent refuge dans la divinité, comme Jaspers et Gabriel Marcel et, d'autre part, les existentialistes athées dont il fait partie et qui estiment que l'existence précède l'essence. C'est sur cette dernière position qu'insiste Sartre pour faire de l'existentialisme un humanisme.

II s'ensuit que l'homme, comme être ouvert à l'existence, fait alors l'expérience du délaissement : "Si l'on entend, suggère Sartre, que quel que soit l'homme qui apparaît, il y un avenir à faire, un avenir vierge qui l'attend, alors ce mot est juste ${ }^{8}$ ". C'est pourquoi, la morale existentialiste se révèle sur ce point comme une morale d'action et 
d'engagement au sein de laquelle l'être humain est à tout instant responsable de tous ses actes mais également responsable de tous les autres hommes, en ce que chacune de ses actions s'inscrit dans une intersubjectivité. L'humanisme d'une telle réflexion réside pour Sartre dans la priorité qu'est donnée à l'homme, priorité dans laquelle celui-ci retrouve une dignité en ce qu'il est l'auteur de sa propre loi et de son propre salut : «il faut que l'homme se retrouve lui-même, et se persuade que rien ne peut le sauver de lui-même ${ }^{9}$.

Ce que condamne Camus dans ce système de pensée, qu'elle prenne Dieu ou l'histoire comme horizons, c'est précisément «le saut hors du problème ${ }^{10}$ ", «l'évasion ${ }^{11}$ ", autrement dit, sa propre négation comme moyen de dépassement. Rappelons que le saut, compris ici comme passage ou rupture, passage sans transition d'une sphère à une autre, est une référence explicite à Kierkegaard. Pris dans une dichotomie entre le fini et l'infini, l'homme ne trouve son salut qu'en effectuant ce saut qualitatif dans la foi, exprimant ainsi l'idée que c'est bien l'irrationnel qui sauve l'homme de sa propre condition'12. Afin de cerner le nœud de cette articulation chez Camus, il est nécessaire de reprendre la notion d'absurde, qui rend compte des termes du problème. Dans Le mythe de Sisyphe, l'auteur développe l'idée que l'absurde est l'état métaphysique de l'homme conscient ${ }^{13}$. Pris entre le désir de savoir, cultivé par la raison, et cette dimension irrationnelle du monde toujours renaissante, l'homme prend conscience à la fois de ses limites et de son refus de s'y résigner. C'est dans cette opposition, cette lutte sans repos, que surgit la conscience de l'absurde : "L'absurde est essentiellement un divorce. II n'est ni dans l'un ni dans l'autre des éléments comparés. II naît de leur confrontation ${ }^{14}$ ", admet Camus. A partir de cette confrontation entre l'action de l'homme et le monde, entre son aspiration et sa condition, Camus confère à la notion d'absurde toute sa densité : la conscience douloureuse d'une contradiction entre des termes que rien ne peut séparer. Puisque c'est bien en cela que l'homme reconnaît son propre univers, il ne s'agit aucunement de vouloir y échapper ou de vouloir fuir, mais bien au contraire «de préserver cela même qui m'écrase, de respecter en conséquence ce que je juge essentiel en lui ${ }^{15}$ ".

C'est pour cela que la question essentielle est de savoir s'il est possible de vivre sans appel avec cette conscience de l'absurde. L'intérêt de la position de Camus est de montrer qu'il ne s'agit guère de trouver une solution à ce conflit, mais de le conserver en le transfigurant. Or, si l'attitude existentielle est définie comme «un 
suicide philosophique ${ }^{16}$ ", c'est parce que chacun de ses penseurs commence par réfléchir en termes de résolution, en opérant une négation rédemptrice de l'obstacle. Parti d'une pensée consciente du non-sens du monde, l'existentialisme recherche "des raisons raisonnantes ${ }^{17}$ » qui lui permettent ainsi de fonder une sens à ce qui n'en a pas, et d'échapper à l'obstacle. C'est ce point qui pose difficulté : c'est l'absurde lui-même qui fait sens, le sens de notre être au monde comme être en conflit et en rupture. Pour vivre, il ne convient pas de donner un sens à ce qui n'a pas, mais de comprendre que c'est ce non-sens qui caractérise la vie humaine. II s'ensuit que l'erreur dans laquelle s'est fourvoyé l'existentialisme réside dans cette exigence de nivellement, autrement dit de faire disparaître un des termes de la tension. Ainsi, le défaut de Chestov pour Camus est de conclure à la vanité de la raison, concluant ainsi à l'irrationalité du monde. Ce besoin de tout justifier, en supprimant la raison, fait du même coup disparaitre cette tension dans laquelle s'enracine l'absurde : "Tout est sacrifié ici à l'irrationnel et l'exigence de clarté étant escamotée, l'absurde disparaît avec un des termes de sa comparaison 18 ". II est vain de nier la raison car l'efficacité de celle-ci consiste précisément à nous confronter à l'irrationnel à travers ce besoin de clarté, d'explication. Bien que la raison soit certes limitée, cela ne doit pas nous conduire pour autant à la nier car elle reste le seul moyen pour faire l'expérience de son contraire : c'est en cela qu'elle a un pouvoir, celui de nous faire prendre conscience de la rupture, de la scission entre l'action humaine et le monde.

En faisant porter tout le poids de la réflexion sur un seul des termes, l'équilibre fondé par la tension entre contraires, disparaît et supprime cette conscience de l'absurde. En effet, cette tension naît de la contradiction entre sl'irrationnel du monde et la nostalgie révoltée ${ }^{19}$ ». Ce point est décisif en ce qu'il est symptomatique du besoin de guérir, de dépasser cette antinomie de la condition humaine. Tout le mouvement existentialiste, bien qu'il rende compte d'un non-sens, se donne pour visée de sortir de cette impasse, de trouver des moyens de ne plus en faire l'expérience, que ce soit par le retour à la divinité ou par la croyance au progrès de l'Histoire. "Le philosophe abstrait et le philosophe religieux partent du même désarroi et se soutiennent dans la même angoisse ${ }^{20}$ ». Cette angoisse est celle du délaissement, cette conscience d'un abandon qui se transforme en une volonté de réconciliation, d'unité, qui en réalité n'est qu'une manière de faire ce saut qui évite l'absurde. Ce n'est pas 
la guérison qu'il faut chercher dans la pensée, c'est l'acceptation de cette tension, c'est finalement vivre avec ses maux. "Cet état d'absurde, poursuit l'auteur, il s'agit d'y vivre. Je sais sur quoi il est fondé, cet esprit et ce monde arc-boutés l'un contre l'autre sans pouvoir s'embrasser ${ }^{21}$ ".

Du coup, une des thèses essentielles de l'existentialisme, la liberté en soi, n'a plus de sens, dans la mesure où elle ne se pose que pour le penseur qui a fait ce saut, et qui par conséquent a substitué à l'absurde une forme d'éternité. La liberté ne peut être posée qu'en termes d'expression car elle se limite à une liberté d'esprit ou d'action. Or, l'attitude existentialiste interprète cette liberté comme libération à l'égard d'un maître ou d'une hiérarchie, alors qu'en réalité elle n'est que la forme ultime d'un esclavage. La raison de la liberté profondément humaine réside dans son contraire, celle qui naît de la lucidité de notre condition, que tout finalisme ne vaut rien devant cette vérité intolérable : "ll n'y a pas de lendemain22". La seule liberté est de décider d'accepter de vivre dans un tel univers tendu par des contradictions et des ruptures, univers dépossédé de valeurs. II ne s'agit pas, une fois conscient de l'absurdité de se demander si cela mérite encore la peine de vivre, mais bien plutôt de se dire que ce qui compte désormais, c'est de "vivre le plus ${ }^{23}$ ", c'est-à-dire de prendre en compte l'unique exception qu'est la mort et de vivre en l'acceptant. C'est ici que réside tout le sens du mythe de Sisyphe : pris dans un condition absurde, Sisyphe reconnaît le non sens de son existence, mais c'est en prenant en compte celui-ci qu'il affirme sa révolte, cet effort pour continuer à vivre an acceptant sa condition.

À partir de ces éléments, il faut reconnaître que Camus, en se démarquant de la pensée existentialiste, s'affirme comme l'héritier d'un autre existentialisme, celui qui dans sa réflexion a su maintenir et exprimer cette tension qui naît de l'absurde. Le défaut de l'existentialisme moderne est d'avoir fait le pas vers un nivellement de cette tension et de l'avoir ainsi escamotée. Le fait que l'auteur ne cesse de convoquer les philosophes tels que Pascal, Kierkegaard, Nietzsche, sert à montrer comment chacun à sa manière, a d'abord saisi cette conscience de l'absurde. Ce qui intéresse Camus, ce sont bien les prémisses de cet existentialisme, à savoir cette conscience du nonsens, du divorce entre ce désir d'infini et notre finitude. Tant que cette tension est maintenue, Camus y donne son assentiment. Le meilleur exemple est sans doute celui de Nietzsche dont Camus reconnaît explicitement sa filiation : "La philosophie de Nietzsche tourne 
certainement autour du problème de la révolte. Exactement, elle commence par être une révolte ${ }^{24}$ ". Cette pensée opère une véritable chirurgie, une transmutation des valeurs en reconnaissant tout d'abord que le monde est dépourvu de finalité et d'unité, ce qui empêche du même coup de le juger. La tension inhérente à l'absurde s'enracine en ce sens dans le tragique du philosophe allemand qui réside dans la vacuité du monde dans lequel Dieu n'existe pas. La seule morale réside dans cette lucidité. C'est en poussant ce nihilisme jusqu'en dans ses dernières conséquences que Nietzsche parvient alors à faire de cette tension une véritable révolte, une joie tragique qui considère la mort de Dieu comme un «fait acquis 25 ». Rappelons : le nihiliste n'est pas celui qui ne croit à rien, mais uniquement celui qui ne croit pas à ce qui est 26 .

Si Camus reconnaît cet autre existentialisme chez Nietzsche, c'est parce que ce dernier ne s'est pas dérobé face à l'absurde, et par conséquent a adhéré au seul principe de morale vraiment créatif : «ll faut accepter l'inacceptable et se tenir à l'intenable27». La liberté s'enracine bien dans cette acceptation de la finitude, dans cette abolition des fins qui permet à l'individu de donner une adhésion pleine et entière à ce monde. C'est ici que la transmutation des valeurs prend tout son sens : remplacer la valeur du juge par celle du créateur. Cette liberté qui s'exprime dans l'affirmation du devenir se présente comme une des prémisses de la liberté chez Camus, en ce que celle-ci ne peut être vécue que sous le mode de la confrontation avec la mort, caractéristique ultime du devenir.

Toute cette analyse nous montre donc que les prémisses de cet existentialisme dont se revendique Camus, sont bien les siennes puisqu'elles font surgir en un même cri la conscience de l'absurdité. Toutefois, cette dernière n'est en aucun cas le stade ultime de sa propre réflexion. Si de fait, il faut admettre que l'existentialisme comme système de pensée est rejeté par Camus, c'est sur le plan de ses conséquences. Ce qui signifie qu'un autre existentialisme serait possible : en conservant la conscience de l'absurde, il faudrait aboutir à une conséquence radicalement distincte et qui pourrait rendre possible une action et une pensée nouvelles. Or, chez Camus, celle-ci ne peut prendre que la forme de la révolte : elle est le mouvement même de la vie et on ne peut la nier sans renoncer soi-même à vivre ${ }^{28}$. En effet, l'absurde est d'abord une tension qui devient opératoire, dynamique, dans la mesure où elle a pour conséquence de produire un effort, vécu à la fois comme un défi et comme une 
conquête : «J'exalte l'homme devant ce qui l'écrase et ma liberté, ma révolte et ma passion se rejoignent alors dans cette tension, cette clairvoyance et cette répétition démesurée 29 ». En ce sens, la réconciliation n'est possible que sous le mode d'une action, d'une force dynamique qui fait tendre l'homme vers son propre dépassement, dépassement qui trouve sa raison d'être dans l'impossibilité même de se réconcilier : «ll faut vivre avec le temps et mourir avec lui30". Cette humaine contradiction devient la condition d'une conquête, celle qui consiste à se surmonter. II ne s'agit surtout pas ici d'occulter la contradiction essentielle qui habite l'homme, mais bien au contraire d'en saisir toute la tension pour envisager un nouveau rapport au monde et à soi-même.

Cet autre existentialisme qui aboutit à la révolte n'a de point commun avec l'existentialisme classique que ses prémisses, ce qui nous conduit alors à reformuler la définition même de la notion. S'il faut reconnaître chez Camus une attitude existentielle, c'est au sens d'une pensée qui accepte la part de malheur de l'existence. Mais c'est cette acceptation qui confère une nouvelle dignité à l'humain. Dans son désespoir, l'homme forge des raisons d'espérer. Ce paradoxe est celui de la révolte car chez Camus, c'est bien la force du désespoir qui fait revenir l'homme à lui-même et à sa propre histoire. Rappelons à ce titre, cette précieuse indication : " ll n'y a pas de soleil sans ombre, et il faut connaître la nuit. L'homme absurde dit oui et son effort n'aura plus de cesse ${ }^{31}$ ". Cette affirmation de la vie n'est donc possible que parce qu'elle se conjugue avec la mort, caractéristique de l'existence humaine. Certes, la vie n'a pas de sens, mais c'est pour cette raison qu'elle crée l'homme car elle lui confère ce qui lui donne sa pleine valeur : celle d'un être qui, confronté à l'absurde, ne se retrouve que dans la lutte. L'existence est au centre même de cette réflexion, mais à la différence des existentialistes, elle est toujours l'objet d'une conscience claire et lucide, sans détournement ni évasion. Autrement dit, la pensée de l'existence pour Camus est une connaissance qui implique l'acceptation des conditions qu'elle impose, et qui fait tendre l'homme vers lui-même : un être, sans subterfuge, qui construit son propre destin.

\author{
Adelino Braz \\ Docteur en philosophie de l'Université de \\ Paris I Panthéon-Sorbonne \\ Rattaché à l'unité de recherche : NoSoPhi \\ (Normes, Société, Philosophie) - Paris I
}




\section{Adelino Braz}

1. Albert Camus, Les Nouvelles littéraires, 15 novembre 1945, dans Essais, «Extraits d'interviews", Paris, Gallimard "Bibliothèque de la Pléiade», 1965, p. 1424. Toutes les références aux essais de Camus sont extraites de cette même édition.

2. Interview à «Servir» du 20 décembre 1945, dans Essais, p. 1428.

3. Le mythe de Sisyphe, dans Essais, p. 165.

4. Jean-Paul Sartre, L'existentialisme est un humanisme, Paris, Nagel, 1970, p. 93.

5. Dernière interview d'Albert Camus, 20 décembre 1959, dans Essais, p. 1926.

6. Jean-Paul Sartre, ibid., p. 89.

7. Leibniz, Examen préalable des objections de Lamy, 30 novembre 1702, Paris, Flammarion, 1994, p. 258.

8. Jean-Paul Sartre, ibid., p. 39.

9. Jean-Paul Sartre, ibid., p. 95.

10. Albert Camus, Le mythe de Sisyphe, p. 1436, note 1 de la p. 122.

11. Ibid., p. 122.

12. Søren Kierkegaard, Traité du désespoir, Paris, Gallimard, 1949, p. 62.

13. Le mythe de Sisyphe, p. 128.

14. Ibid., p. 120.

15. Ibid., p. 121.

16. Ibid., p. 128.

17. Ibid., p. 129.

18. Ibid., p. 125.

19. Ibid., p. 126.

20. Ibid., p. 133.

21. Ibid., p. 128.

22. Ibid., p. 141.

23. Ibid., p. 123.

24. L'homme révolté, dans Essais, p. 477.

25. Ibid., p. 477.

26. Ibid., p. 479.

27. Ibid., p. 481.

28. Ibid., p. 707.

29. Le mythe de Sisyphe, p. 166.

30. Ibid., p. 165.

31. Ibid., p. 198. 